\title{
Making medicine more scientific
}

\author{
Howard Hiatt and Lee Goldman
}

\section{Modern biology has far-reaching Implications for medicine. But a new type of medical training will be necessary if advances in scientific understanding are to become advances In treatment.}

MEDICAL school research and curricula in the United States and elsewhere have been profoundly changed by the revolution in biology. Our understanding of the causes of heart disease, cancer and a range of other illnesses is rapidly deepening, and our capacity to prevent and treat some is improving. Many clinical departments have physicians who are well trained in biology as well as patient care, and who conduct sophisticated basic research while continuing to teach younger colleagues and medical students. Little wonder that the era of scientific medicine is said to have arrived.

\section{Unscientific}

But has it? Many of these same faculty members, through no fault of their own, continue to practise medicine in ways that can hardly be termed scientific. All physicians must cope with inadequate data about the risks and effectiveness of many of the procedures they perform. Many have received little formal training to help evaluate the information that does exist, and are not as well prepared as they might be to help their patients make decisions.

With a few exceptions, the research of academic clinical departments until recently focused almost exclusively on the biology of disease. The resulting delays in applying the so-called 'evaluative clinical sciences' - including statistics, epidemiology, decision analysis, costeffectiveness analysis, health-services research, economics, ethics and computer sciences - have led to major problems in advanced health-care systems. These subjects have so far had little impact on the decisions of individual physicians, and even less on national health policy.

Yet if this movement is strengthened, quality of medical care will be greatly improved. Accurate assessment of the cost-effectiveness of interventions may have important effects on health-care reform. And in time, academic departments will acquire a body of individuals equipped to realize the benefits of these skills in the clinic and in teaching.

Since the Second World War, an appreciation of the far-reaching medical implications of the new biology, combined with generous financial support from the National Institutes of Health, has led to a new climate in the clinical departments of many leading US medical schools. But faculty members and students were not trained to apply such advances to the health of the population. Even population-based studies of medical practice and disease were felt to lie on the periphery of academic medicine. And studies of disease natural history, diagnostic and therapeutic outcomes and systems approaches to the quality of medical care had little or no place on the research agenda. Even such crucial areas as primary care were considered to be intellectually unchallenging and received scant attention. Other important issues, such as the effects of treatments on the quality as well as the length of life, the role of patients and their families in decisions about treatment, the importance of non-physicians in care and the cost of interventions received even less attention.

The medical neglect of the evaluative clinical sciences also worked in the other direction; many key statistical, economic, ethical and other concepts are only now developing in response to medical problems. So for decades biostatisticians and epidemiologists have concentrated largely on public health rather than individual patients.

In the past, the adoption of changes in diagnosis and therapy without rigorous evaluation helped to overwhelm the health-care system with options. Experts to help patients and policymakers select from among worthy choices on a rational basis are essential.

New methods have already markedly improved our capacity to evaluate patients, diseases and their management. The randomized clinical trial, virtually unknown a generation ago, has been refined to the point where tens of thousands of patients in many nations can be studied simultaneously. New statistical approaches often allow results approximating those from trials to be extracted from routine clinical data. And improved measures of health status, cost and quality of life have facilitated research into conditions affecting morbidity.

In the United States, some of these developments have been supported by the federal Agency for Health Care Policy and Research. Yet overall, the funds allocated to such research are but a small fraction of those for more traditional biomedical science.

More than 20 years ago, the Commonwealth Fund, the Robert Wood Johnson Foundation and the Henry J. Kaiser Family Foundation introduced successful training programmes in the social and evaluative clinical sciences for physicians. At Brigham and Women's Hospital, Harvard Medical School and the Harvard School of Public Health, our two-year Program for Training in Research in Clinical Effectiveness has built upon this experience to become one of the largest of its kind. Begun in 1986, it has grown rapidly (and unexpectedly) from 8 participants in 1986 to 89 in 1994.

The programme begins with intensive courses in biostatistics and epidemiology, as well as electives in decision sciences, health services research, health policy, quality improvement and public health. The courses are designed for physicians concerned with patient care rather than traditional public health issues.

All trainees do one or more research projects. Topics have included the costeffectiveness of thrombolysis after acute myocardial infarction, risk factors for the development of kidney stones, a randomized trial of aspirin in angina, the role of history and physical examinations in diagnosing carpal tunnel syndrome, the use of blood cultures in diagnosing bacteraemia and the role of surrogates in decision-making for mentally incompetent patients.

\section{Initial reluctance}

Of the first 69 graduates, 58 (84 per cent) are in academic medicine. Many fellows also take advanced courses at the Harvard School of Public Health. To begin with, many department chairs appeared surprised, even dismayed, that able young colleagues had chosen to pursue 'clinical effectiveness' rather than 'real' (biological) research. Some physicians were compelled to ask that the contents and goals of the programme be explained to their chairs before sponsorship for them was forthcoming.

The enthusiasm and success of the early graduates, however, appear to have increased interest both within their own departments and elsewhere. Several chairs who had originally agreed to sponsor one person with reluctance have themselves now established units in clinical effectiveness.

Howard Hiatt and Lee Goldman are at Brigham and Women's Hospital, 75 Francis Street, Boston, Massachusetts 02115, USA, and professors of medicine at Harvard Medical School, Massachusetts. 